\title{
Analysis of Cognitive Radio Spectrum Access with Optimal Channel Reservation
}

\author{
Xiaorong Zhu, Lianfeng Shen, and Tak-Shing Peter Yum
}

\begin{abstract}
A Markov chain analysis for spectrum access in licensed bands for cognitive radios is presented and forced termination probability, blocking probability and traffic throughput are derived. In addition, a channel reservation scheme for cognitive radio spectrum handoff is proposed. This scheme allows the tradeoff between forced termination and blocking according to QoS requirements. Numerical results show that the proposed scheme can greatly reduce forced termination probability at a slight increase in blocking probability.
\end{abstract}

Index Terms-Cognitive radio networks, spectrum access, channel reservation.

\section{INTRODUCTION}

$\mathbf{R}$ ECENTLY, the technology of cognitive radios has captured the attention of many researchers in that it promises an effective way of enhancing spectrum usage and solving the problem of heterogeneity of radio devices [1-3]. A Markov chain model was proposed to predict the behavior of open spectrum access in unlicensed bands [2]. For spectrum access in licensed bands, two Markov chain models were investigated in [4].

In a radio network consisting of primary users (licensed users) and cognitive users (unlicensed users), the primary users do not always fully utilize their spectrum. The cognitive users may therefore temporally occupy the unused sub-bands. However, the cognitive users need to vacant these sub-bands when primary users want to use them. When this occurs, the affected communication links of the cognitive users will be lost. But if the cognitive users can sense idle sub-bands they can reconstruct the communication links to them. This is called spectrum handoff.

In this letter, we present a general model for cognitive radios access in licensed bands. Based on this, we derive the forced termination probability, the blocking probability and system throughput. Furthermore, we propose the use of channel reservation to tradeoff the forced termination probability and blocking probability. This scheme is similar to channel reservation used in circuit-switched networks [5]. It was shown that significant higher throughput could be achieved if a proper number of channels were reserved.

Manuscript received December 18, 2006. The associate editor coordinating the review of this letter and approving it for publication was Prof. Gianluca Mazzini. This work was supported by the Natural Science Foundation of China (No. 60472053) and UGC (University Grant Council)/AoEIT (Area of Excellence in Information Technology) of Hong Kong.

$X$. Zhu and L. Shen are with the National Mobile Communications Research Laboratory, Southeast University, Nanjing, China (e-mail: zxr@semit.com.cn, lfshen@seu.edu.cn).

T.-S. P. Yum is with the Department of Information Engineering, The Chinese University of Hong Kong, China (e-mail: tsyum@ie.cuhk.edu.hk).

Digital Object Identifier 10.1109/LCOMM.2007.062058.

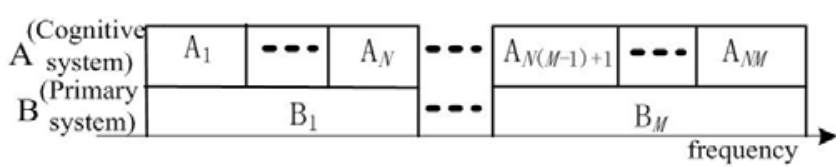

Fig. 1. Frequency bands used by two types of radio systems.

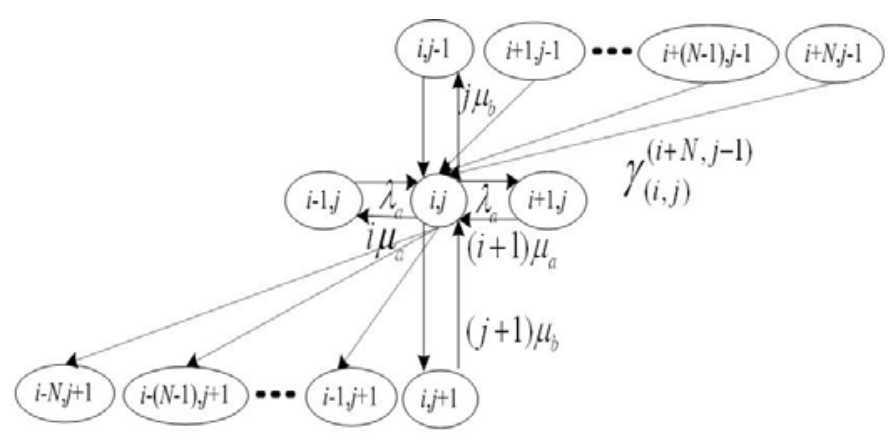

Fig. 2. Markov chain cognitive radio without spectrum handoff.

\section{SySTEM MODELING}

Let there be two types of radio users, the primary users and cognitive users, operating in the same spectrum. The spectrum consists of $M$ primary bands and each primary band is divided into $N$ sub-bands. The cognitive users can use channels $A_{1}$ to $A_{N M}$ while the primary users can use channels $B_{1}$ through $B_{M}$. The A and B channels overlap with each other, as indicated in Fig.1. The primary users have the priority to use the spectrum and can reclaim any sub-bands temporarily used by cognitive users. Therefore, the presence of cognitive users is entirely transparent to the primary users.

\section{Cognitive Radio Without Spectrum HandofF}

The process of spectrum occupation is modeled as a continuous time Markov chain. It is characterized by its states and transition rates. The $N M$ sub-bands are shared by the primary users and cognitive users. In this case states are described by an integer pair $(i, j)$, where $i$ is the total number of sub-bands used by cognitive users and $j$ is the total number of primary bands used by the primary users. We assume the arrivals of cognitive users and primary users are both Poisson processes with arrival rates $\lambda_{a}$ and $\lambda_{b}$. The corresponding service times are exponentially distributed with rates $\mu_{a}$ and $\mu_{b}$.

As the primary users have the priority to use the spectrum, the cognitive users can be preempted by primary users. Depending on the number of sub-bands occupied by the cognitive users in the newly preempted primary band, a forced termination in state $(i, j)$ will move the state to one of $(i, j+1),(i-1, j+1),(i-2, j+1), \ldots,(i-(N-1), j+1)$, and 


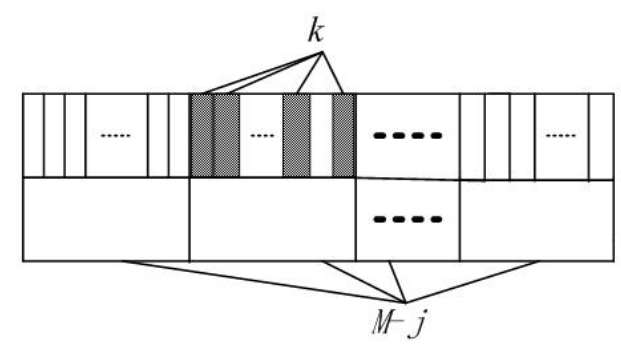

Fig. 3. Sub-bands occupied in the forced termination state.

$(i-N, j+1)$ as shown in Fig.2. The transition rate of a forced termination depends on the number of sub-bands $k$ used by the cognitive users in this primary band. Let $\gamma_{(i-k, j+1)}^{(i, j)}$ denote the transition rate from state $(i, j)$ to state $(i-k, j+1)$. This transition occurs when the $k$ sub-bands are in the same primary band while the residual $(i-k)$ sub-bands are distributed in the other $(M-j-1)$ primary bands as shown in Fig.3. This is the classical partition problem in probability and we can readily obtain

$\gamma_{(i-k, j+1)}^{(i, j)}=\frac{\left[\begin{array}{c}N \\ k\end{array}\right]\left[\begin{array}{c}(M-j-1) N \\ i-k\end{array}\right]}{\left[\begin{array}{c}(M-j) N \\ i\end{array}\right]} \lambda_{b} \quad k=1,2, \ldots, N$

Let $\Phi$ denote the set of feasible states of the Markov chain shown in Fig. 2 and $\phi(i, j)$ be an indicator function of $\Phi: \phi(i, j)$ $=1$ if $(i, j) \in \Phi$, and 0 otherwise.

Let $P(i, j)$ be the state probability. From Fig.2, the set of balance equations can be written as

$$
\begin{aligned}
& {\left[j \mu_{b}+i \mu_{a}+\lambda_{a}+\sum_{k=0}^{N} \gamma_{(i-k, j+1)}^{(i, j)}\right] P(i, j) \phi(i, j)=} \\
& \lambda_{a} P(i-1, j) \phi(i-1, j) \\
& +(j+1) \mu_{b} P(i, j+1) \phi(i, j+1) \\
& +(i+1) \mu_{a} P(i+1, j) \phi(i+1, j) \\
& +\sum_{k=0}^{N} \gamma_{(i, j)}^{(i+k, j-1)} P(i+k, j-1) \phi(i+k, j-1) \\
& \sum_{i=0}^{N M} \sum_{j=0}^{M} P(i, j) \phi(i, j)=1
\end{aligned}
$$

where $i=0,1, \ldots, N M$ and $j=0,1, \ldots, M$. The total number of states is $1+\sum_{i=1}^{M}(i N+1) \approx N M^{2} / 2$.

Forced termination represents a disruption of service and should be kept below a tolerable level. When the state transition is from state $(i, j)$ to state $(i-k, j+1), k$ out of $i$ cognitive users will experience forced termination. Therefore, forced termination probability, $P_{F}$, is

$$
P_{F}=\sum_{i=0}^{N M} \sum_{j=0}^{M} \sum_{k=1}^{N} \frac{k}{i} \gamma_{(i-k, j+1)}^{(i, j)} P(i, j)
$$

\section{Cognitive Radio with Spectrum Handoff}

If spectrum handoff is allowed, preempted cognitive radio calls will moved immediately to idle sub-bands elsewhere. This re-allocation of band can either be performed by the

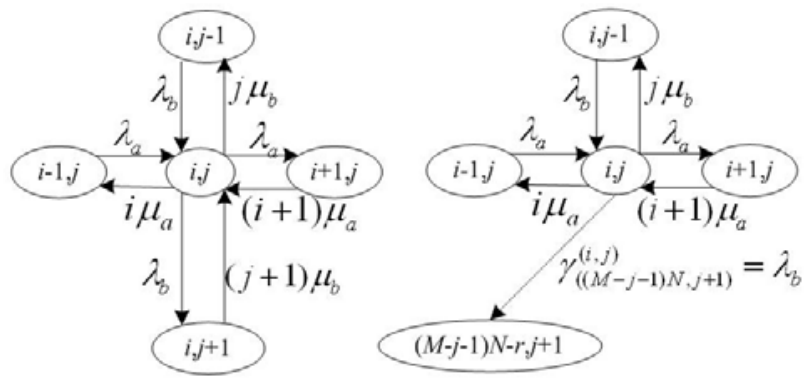

(a) if $i+N j \leq N(M-1) \quad$ (b) if $N(M-1)<i+N j \leq N M-r$

Fig. 4. Rate diagram of state $(i, j)$ with spectrum handoff and channel reservation.

base station centrally or by the cognitive radios through a suitable distributed protocol. Therefore, as long as there are idle sub-bands around forced termination will not occur. Thus for state $(i, j)$, if $i+j M \leq(N-1) M$, forced termination will not occur with the arrival of a primary user; otherwise, forced termination(s) will move state $(i, j)$ to state $((M-j-$ 1) $N, j+1)$ with transition rate

$$
\gamma_{((M-j-1) N, j+1)}^{(i, j)}=\lambda_{b}
$$

\section{Optimal Channel Reservation}

In this section, we study the use of channel reservation to reduce the probability of forced termination. Let $r$ be the number of sub-bands reserved for spectrum handoff. ( $r=0$ corresponds to channel reservation not used) Fig.4 shows the transition rate diagram of state $(i, j)$ with $r$ reserved subbands. The blocking of cognitive users occurs when the current bandwidth occupancy $(i+N j)$ plus $r$ equals to the total bandwidth $N M$. Let $P_{B}(r)$ denote the blocking probability with $r$ reserved channels. We can readily obtain

$$
P_{B}(r)=\sum_{i=0}^{N M-r} \sum_{j=0}^{M} \delta(i+N j+r-N M) P(i, j)
$$

With $r$ reserved sub-bands, the forced termination probability becomes

$P_{F}(r)=\sum_{i=0}^{N M-r} \sum_{j=0}^{M} \frac{k}{i} \gamma_{(i-k, j+1)}^{(i, j)} \delta(i+N j>(M-1) N) P(i, j)$

From (6) and (7), $P_{B}(r)$ and $P_{F}(r)$ can be tradeoff by adjusting $r$ according to the QoS requirements.

A natural way of choosing the optimal $r$ is to maximize the throughput $\rho(r)$ of cognitive users, where throughput is defined as the average number of service completions per second, namely

$$
\rho(r)=\sum_{i=1}^{N M-r} \sum_{j=0}^{M} P(i, j) \cdot i \mu_{a}
$$

The optimal $r$ can easily be obtained by enumeration. 


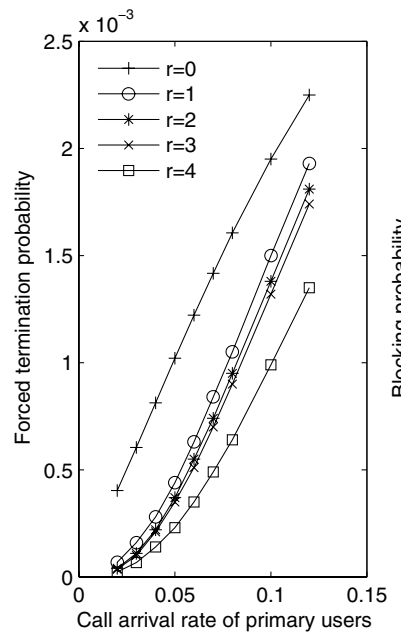

(a)

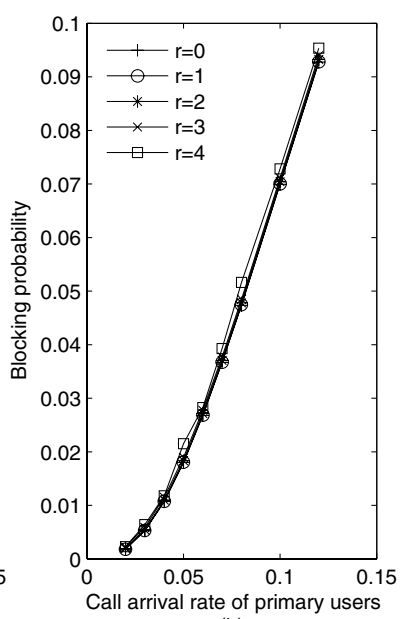

(b)
Fig. 5. The tradeoff between forced termination probability and blocking probability for $r$ varying from 0 to 4 , where $\mu_{a}=0.82, \lambda_{a}=0.68$ and $\mu_{b}=0.06$.

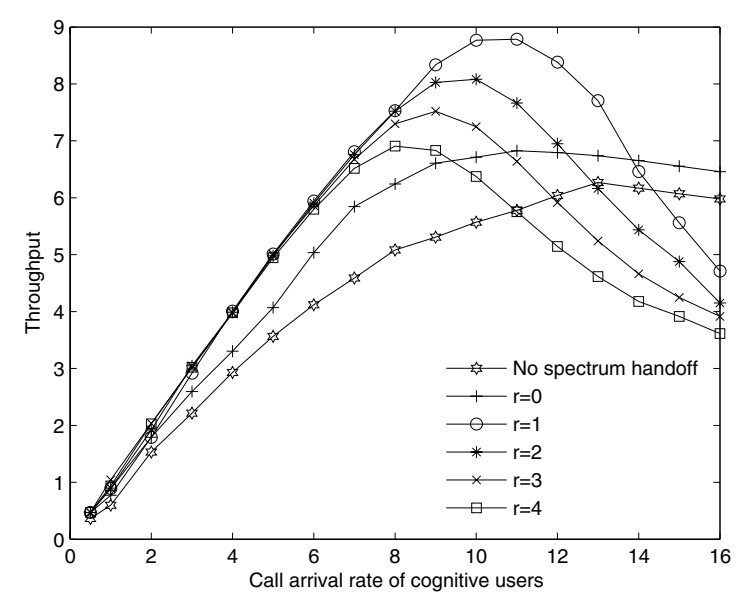

Fig. 6. Traffic throughput with different call arrival rate of cognitive users, where $\mu_{a}=0.8, \mu_{b}=0.06$ and $\lambda_{b}=0.08$.

\section{PERformance EVAluation}

To evaluate the performance of spectrum handoff and channel reservation consider a system with $M=3$ primary bands and $N=6$ sub-bands in each primary bands. Fig. 5 shows the forced termination probability and blocking probability for various values of $r$ as a function of primary users call arrival rate. We see that channel reservation can significantly reduce forced termination probability at a very small increase of new call blocking probability.

Fig.6 shows the throughput of cognitive users. It is seen from this case that spectrum handoff and channel reservation can significantly increase throughput. The optimal value $r^{*}=1$ should be used for $\lambda_{a}<14$ and $r^{*}=0$ for $\lambda_{a} \geq 14$. Fig.7 (a) and $(b)$ show the optimum numbers of reserved sub-bands that maximize the throughput of cognitive users for $\lambda_{b}=0.04$ and $\lambda_{b}=0.12$ respectively. It is seen that when the traffic rates of cognitive users are smaller, more sub-bands should be used for reservation.
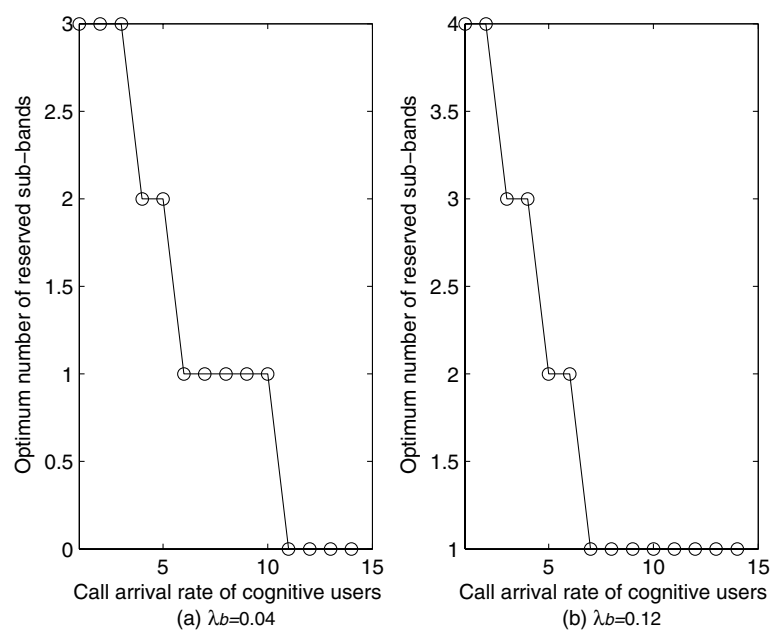

Fig. 7. The optimum number of reserved sub-channels, where $\mu_{a}=0.8$ and $\mu_{b}=0.06$.

\section{CONCLUSION}

We have shown by Markov chain analysis that channel reservation can significantly increase the throughput of cognitive radio users.

\section{REFERENCES}

[1] I. F. Akyildiz, W.-Y. Lee, M. C. Vuran, and S. Mohantly, "Next generation/dynamic spectrum access/cognitivw radio wireless network: a survey," available:www.sciencedirect.com.

[2] Y. Xing, R. Chanddramouli, S. Mangold, and S. Shankar, "Dynamic spectrum access in open spectrum wireless networks," IEEE J. Sel. Areas Commun., vol. 24, pp. 626-636, Mar. 2006.

[3] T. Weiss and F. Jondral, "Spectrum pooling: an innovative strategy for the enhancement of spectrum efficiency," IEEE Commun. Mag., vol. 42, pp. S8-S14, Mar. 2004.

[4] F. Capar et al., "Comparison of bandwidth utilization for controlled and uncontrolled channel assignment in a spectrum pooling system," in Proc. VTC Spring 2002.

[5] K.-M. Chan and T.-S. P. Yum, "The maximum mean time to blocking routing in circuit-switched networks," IEEE J. Sel. Areas Commun., vol. 12, no. 2, pp. 313-321, Feb. 1994, 